\title{
For a Scientific Biosemiotics
}

\section{Marcello Barbieri}

Received: 21 March 2009 / Accepted: 10 April 2009 /

Published online: 6 May 2009

(C) Springer Science + Business Media B.V. 2009

In January 2009, our Journal celebrated its first year and I asked all members of the Editorial Team to take stock of the situation, describe the problems that biosemiotics is facing at the moment, and send their suggestions for the future. After a first round of comments, inspired by the successful launch of the journal, the consultation quickly turned into a discussion about the very nature of our field. Biosemiotics is a synthesis of biology and semiotics, but is it more than the sum of the two disciplines? does it have an 'identity' of its own? and, above all, is biosemiotics a science? These are crucial points for our discipline and soon the discussion focused upon three main questions:

(1) is biosemiotics based on testable models?

(2) does it have an experimental field of its own? and

(3) does it tell us something new about the history of life?

The answers to these questions concern all people who are interested in the life sciences, and this is why I am re-proposing them here. We already have decisive arguments in favour of a scientific biosemiotics, so let us bring them before a wider public, for everybody to see.

(1) Biosemiotics is based on testable models.

It is widely accepted that the distinctive feature of science is the fact that its ideas can be tested. According to Karl Popper, no amount of evidence can prove a statement true, but a single experiment can prove it false. That is why we say that an idea, a model or a theory is scientific if it is falsifiable. It has been suggested however that this is not a completely safe criterion because it is always possible to make a theory immune to falsification by ad hoc hypotheses. The most famous example is the theory of evolution, where it seems that any experimental result can be made to fit the theory with convenient assumptions. Karl Popper himself fell into this trap, once,

M. Barbieri $(\bowtie)$

Dipartimento di Morfologia ed Embriologia, Via Fossato di Mortara 64, 44121 Ferrara, Italy

e-mail: brr@unife.it 
and concluded that evolution is not falsifiable, but that was only a temporary slip and he quickly disavowed it. He pointed out that the discovery of a single human skull in Precambrian strata would immediately falsify the theory of evolution, so this is testable and therefore scientific.

The idea that science is based on testable models, in short, is still the best criterion we have, and it is this criterion that allows us to say that biosemiotics is a science. Its foundational principle is Tom Sebeok's idea that "life and semiosis are coextensive", and this is indeed a falsifiable statement. It implies that the origin of life was also the origin of semiosis so it would be enough to find a single example of semiosis in the inanimate world and the principle would be falsified. Another example is the principle that protein synthesis is a manufacturing semiosis because it is necessarily based on a genetic code. It would be enough to show that proteins (i.e. polypeptides with specific sequences) can be manufactured by living systems without a genetic code, and this principle would be dead. The ideas and the models of biosemiotics, in short, are as testable as those of any other scientific field, and this tells us that they do belong to science.

(2) Biosemiotics has an experimental field of its own.

The fact that biosemiotics consists of testable models, proves that it is a scientific field, but not that it is a 'new' or a 'distinct' field of research. This conclusion is justified only if there is a whole set of biological phenomena that exist in nature and are not accounted for by any other theory. Which is precisely what we do have in our case. The discovery of the genetic code came as a complete surprise exactly because the concept of code did not fit in the official framework of biology. And that was only the beginning. The genetic code was discovered because it was found that protein synthesis is based on adaptors, and these are the molecular fingerprints of any organic code. The presence of adaptors in other biological processes has proved that there are many other organic codes in nature, and these discoveries have started bringing to light a whole new dimension of life. Biosemiotics does have an experimental field of its own because it is the study of organic codes and signs, and these are fundamental biological entities that can no longer be ignored.

(3) Biosemiotics tells us something new about the history of life.

The genetic code appeared at the origin of life and the cultural codes arrived at the end of life's history. According to modern biology there has been no other code in between, and this implies that four billion years of evolution went by without bringing any new codes in existence. The discovery of the organic codes, however, is revealing a completely different scenario. The history of life has been teeming with codes and it is becoming increasingly clear that the process of coding is as fundamental to life as the process of copying is to heredity. More than that. The origin of new organic codes is closely associated with the great steps of macroevolution, and this relationship has extraordinary implications. It suggests that it was new organic codes that gave origin to the major transitions of evolution. A living world full of organic codes, in short, does not change only our description of the history of life. It makes us think again about the logic and the mechanisms of that history. This is what biosemiotics is really about. 
(4) Towards a unified science

There is one more point of the first year consultation that deserves to be brought to the attention of a wider public. We realize that biosemiotics is a science, but does it have a chance of becoming a unified science in the near future? This is an important point because biosemiotics has been developed independently between the 1960s and the 1990s and is still divided into different schools. It is like a new land to which people have come from different countries, speaking different languages and bringing with them different values.

I have been interested in the history of our field for many years, and I had already put together a draft entitled A Short History of Biosemiotics, which described the conditions for a unified science. The simple act of laying down the events in their historical sequence suggested the next logical step in the sequence, and that convinced me that the history of biosemiotics is the key to overcoming the present divisions. This is why I circulated a copy of the draft to all members of the Editorial Team and asked them to send their comments for the final version of the paper.

That started a second round of consultations, and the result is the Short History that is appearing in the present issue. It is far from perfect, but it has been examined by all members of the Editorial Team and almost all their suggestions are now present in the final version. It is therefore a first step towards a shared history and a shared history, in turn, is a first step towards a unified science. The real purpose of $A$ Short History of Biosemiotics is precisely that: to reconstruct the past in order to solve the problems of the present. To look back in order to go forward. 